\title{
Evaluación de un programa piloto de promoción de la salud sexual para personas con diagnóstico de trastorno mental crónico
}

\author{
Andrés López de la Llave ${ }^{1}$, María Castaño ${ }^{2}$ y M. Carmen Pérez-Llantada ${ }^{1}$ \\ ${ }^{1}$ Universidad Nacional de Educación a Distancia, Madrid, España \\ ${ }^{2}$ Fundación INTRAS, Toro, Zamora, España
}

A test of a pilot program for sexual health promotion for people diagnosed with a chronic mental disorder

\begin{abstract}
The goals of this study were the following: a) to explore sexual attitudes and the level of sexual knowledge in people with and without a chronic mental disorder diagnosis, and b) to examine the efficacy of a program aimed at promoting sexually healthy attitudes and increasing knowledge about sexuality. Fifty-three people participated in the study (23 with and 30 without a mental illness diagnosis). Participants diagnosed with a mental illness were assigned to two groups (13 participated in the program and 10 were put on the waiting list). People with a mental illness diagnosis showed healthier sexual attitudes $(t=-5.535, p<.001)$ but people without this diagnosis achieved better results in adequate knowledge about sexuality $(t=-2.057, p<.05)$. The program showed positive effects on the two target variables (sexual knowledge and attitudes) as those who participated in the program showed more knowledge and healthier attitudes.
\end{abstract}

Keywords: Sexual attitudes; schizophrenia; promoting sexual health; mental disorder.

Resumen: Los objetivos de este estudio fueron: a) explorar las actitudes sexuales y el nivel de conocimientos sexuales, en personas con y sin un diagnóstico de trastorno mental crónico, y b) examinar un programa para fomentar actitudes sexualmente saludables e incrementar los conocimientos sobre sexualidad. Participaron 53 personas (23 con diagnóstico de enfermedad mental y 30 sin este diagnóstico). Las personas con diagnóstico de enfermedad mental (PCDEM) se asignaron a dos grupos (13: asistencia al programa y 10: lista de espera). Las PCDEM mostraron actitudes sexuales más saludables $(t=-5.535 ; p<.001)$ pero las personas sin este diagnóstico obtuvieron mejores resultados en conocimientos adecuados sobre sexualidad $(t=-2.057 ; p<.05)$. El programa aplicado mostró efectos positivos en las dos variables objetivo (conocimientos y actitudes sexuales), siendo el grupo de participantes en el programa el que mostró más conocimientos y actitudes más saludables.

Palabras clave: Actitudes sexuales; esquizofrenia; promoción de la salud sexual; trastorno mental.

\section{Introducción}

La Organización Mundial de la Salud (OMS) y la Organización Panamericana de la Salud (OPS), definen el concepto sexualidad como una dimensión fundamental

Recibido: 16 de junio 2020; aceptado: 22 de abril 2021.

Correspondencia: Andrés López de la Llave, Facultad de Psicología, Universidad Nacional de Educación a Distancia, Juan del Rosal, 10, 28040 Madrid, España. Correo-e: alopez@psi.uned.es de la persona que, basada en el sexo, incluye el género, las identidades de sexo y género, la orientación sexual, el erotismo, el vínculo emocional, el amor, y la reproducción (OMS, 2020). En la sexualidad de las personas confluyen factores biológicos, psicológicos, socioeconómicos, culturales, éticos y religiosos. Las personas experimentan y expresan su sexualidad en los planos cognitivo, emocional y conativo. La dimensión sexual resulta determinante también en los aspectos sociales de la vida de las personas. Según la OMS (2020) la salud sexual es 
un estado de bienestar físico, mental y social en relación con la sexualidad. Requiere un enfoque positivo y respetuoso de la sexualidad y de las relaciones sexuales, así como la posibilidad de tener experiencias sexuales placenteras y seguras, libres de toda coacción, discriminación y violencia. Para poder alcanzar altos niveles en salud sexual es necesario que se reconozcan y se garanticen los derechos sexuales de las personas. Un aspecto novedoso y fundamental en esta definición es el referente a los derechos sexuales, considerados universales, y que se fundamentan en la libertad, dignidad e igualdad (Pérez-Conchillo, 2019).

La educación sexual es un término habitualmente reservado a la actuación en poblaciones de niños y adolescentes, y que se lleva a cabo en el ámbito escolar (p. ej. Morales et al., 2018). Pero también se ha señalado que las técnicas educativas se pueden considerar como una herramienta fundamental cuando se implantan programas de promoción de la salud sexual (López de la Llave, 2019; Pérez et al., 2006). Estos programas tienen como objetivo fundamental fomentar el desarrollo de una sexualidad positiva que contribuya al bienestar de las personas (López de la Llave, 2016).

En consonancia con la teoría de la acción planeada (Fishbein y Ajzen, 2011), la experiencia positiva de la propia sexualidad parece estar directamente relacionada con las actitudes. Se ha señalado el efecto mediador de las actitudes hacia comportamientos sexuales en la relación entre el sexo y el número de comportamientos sexuales realizados. Por ejemplo, se ha encontrado que las actitudes hacia la sexualidad correlacionan de forma positiva con el número de comportamientos sexuales realizados y que si deseamos influir en determinados comportamientos sexuales nos deberíamos centrar en las actitudes hacia dichos comportamientos (Blanc, 2020). Por otro lado, desde hace más de tres décadas se ha señalado la importancia de la educación y la adquisición de conocimientos en la generación de actitudes y la posterior manifestación de conductas saludables $(\mathrm{Ca}-$ rrera et al., 2007; Costa y López, 1996 López de la Llave et al., 2015).

En otro orden de cosas, el concepto de «enfermedad mental grave y prolongada» hace referencia a personas diagnosticadas de trastornos psiquiátricos que cursan con un importante deterioro en el funcionamiento social, laboral y personal, que se mantiene en el tiempo, y van acompañados de cierto grado de discapacidad, que impide el desarrollo de una vida normalizada y autónoma. Algunos ejemplos son los trastornos del espectro de la esquizofrenia, trastornos de personalidad, trastornos bipolares...etc. (Goldman et al., 1981). La atención profesional que se presta a esta población se ha dirigido, principalmente, a actuar sobre la sintomatología más llamativa (como puede ser los delirios, las alucinaciones, el lenguaje y comportamiento desorganizado, etc.) que es la que les aleja de los estándares comportamentales normalizados de nuestra sociedad. La atención a su sexualidad en la mayoría de las situaciones es poco abordada, o queda relegada a un segundo plano (Huguelet et al., 2015).

Se puede pensar que esta falta de atención a la sexualidad no significa que sea una dimensión que no deba ser considerada. Algunos estudios sobre conductas de riesgo sexual en personas con diagnóstico de enfermedad mental (PCDEM) han mostrado que este colectivo se involucra de manera más frecuente en conductas de alto riesgo para su salud (Higgins et al., 2006). Las personas con problemas de salud mental son más vulnerables ante abusos o explotación sexual, probablemente debido a que con frecuencia presentan déficits en habilidades de comunicación, tanto como para pedir ayuda, como para negarse a cualquier práctica no deseada o negociar relaciones más seguras (Susser et al., 1993; Susser et al., 1995). Para casi el $75 \%$ de personas con enfermedad mental la vida sexual es importante, sin embargo, solamente un $25 \%$ de ellas manifiesta sentirse sexualmente satisfechas (Navarro et al., 2003). Bonfils et al. (2015) han señalado que las personas con enfermedad mental grave y prolongada indican la importancia que tiene el estigma social de su enfermedad en relación con sus comportamientos sexuales. Estas circunstancias determinan que muchas PCDEM piensen que, a la hora de elegir una pareja sexual o iniciar una relación sexual, sus oportunidades son menores que las del resto de la población. Además, es frecuente que su autoestima esté afectada de forma negativa, considerándose menos atractivas que el resto de las personas, $y$, más específicamente, menos atractivas como pareja erótica debido a su enfermedad mental (Wainberg et al., 2007; Wright et al., 2007).

Los temas más abordados en los estudios que se llevan a cabo sobre este asunto incluyen aspectos relacionados con prevención de infecciones transmisión sexual (60\%), abusos sexuales $(20 \%)$ y educación para la salud sexual $(20 \%)$. Los profesionales que trabajan con este colectivo indican que esta laguna, sobre la salud sexual en personas con enfermedad mental, se podría explicar debido a la creencia de que estos pacientes carecen de sexualidad y son considerados como «asexuales» (Test y Berlin, 1981; Walsh et al., 2014). Sin embargo, otras investigaciones contradicen esta idea, señalando que las personas con enfermedad mental grave y prolongada consideran que la sexualidad es una dimensión muy importante en su vida (McCann, 2010; Raisi et al., 2018; Werner, 2012). 
Hemos encontrado pocos los estudios que tratan específicamente sobre intervención, o implantación de programas de promoción de la salud sexual, en personas con trastornos mentales (Corbett et al., 2017; Pandor et al., 2015). La mayoría de los trabajos que prestan atención a la dimensión sexual de estas personas se centran en la evaluación de diferentes aspectos de la salud sexual. Por ejemplo, el estado de salud sexual en relación con las disfunciones sexuales producidas por antidepresivos (Baldwin y Foong, 2013; Serretti y Chiesa, 2011; Williams et al., 2010) o antipsicóticos (Serretti y Chiesa, 2011), cuestiones relativas a conductas sexuales de riesgo en personas con enfermedad mental (Brown et al., 2010; Higgins et al., 2006; Meade, 2006) y la evaluación de la salud sexual relacionada con infecciones de transmisión sexual (Brown et al., 2010; Khan et al., 2009). La necesidad de programas de educación para la salud sexual en personas con malestar psíquico es un tema relativamente reciente en la literatura (Ford et al., 2003). En este sentido, los programas que se han presentado se centran, casi de forma exclusiva, en una dimensión concreta: las infecciones de transmisión sexual, y sobre todo en lo relativo al VIH (Higgins et al., 2006). En resumen, las escasas intervenciones publicadas sobre este asunto se centran en alguno de los diversos aspectos que conforman la dimensión sexual humana, obviando una visión global e integradora de la sexualidad.

En el caso de la población sin diagnóstico de trastorno mental, las intervenciones que se llevan a cabo para promover la salud sexual ofrecen un contenido también muy variado, centrándose, sobre todo entre los jóvenes, en temas de prevención de Infecciones de Transmisión Sexual (ITS) y de embarazos no deseados (Landry et al., 2003; Kirby, 2011), siendo cada vez es más frecuente la atención a los aspectos actitudinales (Carrera et al., 2007).

Según los datos disponibles, aunque se dan diferencias en cuanto a su sexualidad entre las personas sanas y personas con trastorno mental crónico, no parece suficientemente establecido si estas diferencias son de tal magnitud que requieran diferentes tipos de intervenciones para promover su salud sexual, o si un mismo programa de promoción de la salud sexual pueden resultar igualmente útil en ambos tipos de personas.

Habida cuenta de todo lo anterior, se planteó la exploración de posibles diferencias en salud sexual entre personas que padecen una enfermedad mental (PCDEM) y personas sin diagnóstico de enfermedad mental (PSCEM), más concretamente en su nivel de conocimientos sobre sexualidad y en sus actitudes hacia la sexualidad. Además, se diseñó un programa piloto de «Promoción de la Salud Sexual para personas con enfermedad mental grave», que tratara de contribuir a suplir las carencias observadas y evaluar su eficacia, tras aplicarlo a un grupo de PCDEM

Es por ello que este trabajo tiene dos objetivos generales: a) explorar si existen diferencias en conocimientos sobre sexualidad y en actitudes hacia la sexualidad entre personas con enfermedad mental grave y prolongada y aquellas que carecen de un diagnóstico de enfermedad mental y b) evaluar si tras la implantación de un Programa piloto de Promoción de la Salud sexual, dirigido a personas con enfermedad mental grave y prolongada, los participantes incrementan sus conocimientos sobre sexualidad y mejoran sus actitudes positivas hacia la sexualidad.

\section{Método}

\section{Participantes}

En este estudio participaron un total de cincuenta y tres personas adultas $(N=53)$. Treinta no cumplían con un diagnóstico de trastorno mental según los criterios del DSM-5 (APA, 2013), y no eran residentes en el centro de rehabilitación psicosocial donde se aplicó el programa; este grupo, $n_{1}=30$, lo conformaban 16 mujeres y 14 varones, de entre 20 y 61 años. Los criterios de inclusión: a) ser de nacionalidad española y mayor de edad y) aceptar cumplimentar un cuestionario.

Además, participaron 23 usuarios de un Centro de Rehabilitación Psicosocial (CRPS), que cumplían los criterios para un diagnóstico de trastorno mental grave y prolongado (17 con diagnóstico de esquizofrenia y 6 otros trastornos psiquiátricos, según los criterios del DSM 5); este grupo, $n_{2}=23$, estaba compuesto por 8 mujeres y 15 varones, de entre 19 y 60 años. Los criterios de inclusión de los residentes en el CRPS fueron: a) ser mayor de edad; b) haber mostrado interés o preocupación por temas relacionados con la sexualidad en algún momento desde su admisión al centro; c) no estar tomando medicación que interfiera con su participación en el programa y d) manifestar su deseo de participar tras haber recibido la información sobre los objetivos, contenidos y duración del programa (consentimiento informado).

Se comprobó la ausencia de diferencias estadísticamente significativas en la composición de la muestra en cuanto al género de los participantes, mediante la prueba de Chi cuadrado $\left(\mathrm{Chi}_{(\mathrm{g}, 1=1)}^{2}=1.80, \mathrm{~ns}\right)$. Tampoco se encontraron diferencias estadísticamente significativas en la edad de los participantes ( $\mathrm{U}$ de Mann-Whitney = 286.00, $Z=-1.060, \mathrm{~ns})$. 


\section{Diseño}

Para alcanzar el primero de los objetivos planeados, se utilizó un diseño ex-post-facto con dos muestras independientes. Se recogieron las puntuaciones obtenidas de las variables dependientes (conocimientos de sexualidad y actitudes sexuales) en las dos submuestras para, posteriormente, observar si existían diferencias, en estas variables, entre las personas con diagnóstico de enfermedad mental (PCDEM) y las personas sin diagnóstico de enfermedad mental (PSDEM).

El segundo estudio, que trata de la evaluación de un programa piloto de promoción de la salud sexual, se utilizó un diseño cuasi-experimental con grupo de control y medidas repetidas en la condición «asistencia al programa» (pretest-postest), en el cual, en un primer momento, se recogieron las medidas pretratamiento de las variables dependientes (conocimientos de sexualidad y actitudes sexuales) y posteriormente, una vez desarrollado el programa, se volvieron tomar las medidas de estas mismas variables. Se comprobó la ausencia de diferencias estadísticamente significativas en la composición de los grupos que participaron en el programa en cuanto al género de los participantes, mediante la prueba de Chi cuadrado $\left(\mathrm{Chi}_{(\mathrm{g} . \mathrm{l}=1)}^{2}=0.06, \mathrm{~ns}\right)$. Tampoco se encontraron diferencias estadísticamente significativas en la edad de los participantes ( $\mathrm{U}$ de Mann-Whitney $=62.500 ; Z=$ $-0.155, \mathrm{~ns})$.

\section{Instrumentos}

Dado que el principal objetivo era evaluar la posible utilidad y eficacia de un programa piloto de promoción de la salud sexual en personas con enfermedad mental grave y prolongada, y no se contemplaba la comparación con los resultados de otras investigaciones, se optó por elaborar unos instrumentos de medida que resultaran apropiados al objetivo y además fueran de aplicación sencilla e intelectualmente accesibles a nuestra población diana.

Se utilizaron dos instrumentos de medida, elaborados ad hoc, para medir las dos variables de interés: 1) conocimientos sobre sexualidad y 2) actitudes sexuales.

Cuestionario sobre Conocimientos de Sexualidad (CCS). Este instrumento constaba de 30 ítems dicotómicos (verdadero-falso) que trataban sobre los conocimientos relativos a diferentes áreas de la sexualidad humana. Los contenidos fueron elaborados a partir de las propuestas de cinco expertos (profesionales de la salud mental) e incluía seis áreas de información/conocimientos sobre este tema. Las áreas de conocimiento a las que atendían eran: a) mitos y falacias sexuales, b) conciencia corporal, c) respuesta sexual humana y disfunciones sexuales, d) sustancias y medicamentos, e) infecciones de transmisión sexual y f) métodos anticonceptivos. Las puntuaciones de este cuestionario podían oscilar entre 0 y 10, donde una mayor puntuación indicaría más conocimiento sobre las diferentes áreas de la sexualidad consideradas. La fiabilidad de la prueba presenta un alfa de Cronbach de .85 (Ver apéndice A).

Cuestionario de Opiniones sobre Sexualidad (COS). Partiendo de la escala 28 ítems-ATSS (Diéguez et al., 2005) basada Escala de Actitudes hacia la Sexualidad (ATSS), de Fisher y Hall (1988), se construyó un instrumento con 13 ítems. Las cuestiones se respondían en una escala Likert $(1=$ Nada de acuerdo; 2 = Poco de acuerdo: $3=$ Indeciso; $4=$ De acuerdo; $5=$ Muy de acuerdo). Una puntuación más cercana a uno correspondía a una actitud más positiva, liberal o erotofílica (Fisher et al., 1988) mientras que una puntuación cercana a 5 hacía referencia a una actitud negativa (conservadora o erotofóbica). De los 13 ítems, 10 estaban formulados de manera directa (donde a puntuaciones más bajas hacían referencia a una actitud más positiva, mientras que puntuaciones cercanas a 5 señalarían actitudes más conservadoras. Los restantes se formularon de manera inversa (puntuaciones bajas corresponderían a actitudes negativas y puntuaciones altas a una actitud más liberal). Los ítems inversos fueron transformados a directos para obtener una adecuada interpretación. Para esta prueba se encontrón un índice de fiabilidad (alfa de Cronbach) de .74 (Ver apéndice B).

\section{Procedimiento}

La muestra se obtuvo mediante dos procedimientos, en ambos casos de la misma ciudad del centro de España, y en ningún caso se ofreció compensación económica o de otro tipo por participar. Una parte de los participantes $\left(\mathrm{n}_{1}\right)$ eran personas sin diagnóstico de trastorno mental (PSDEM) que fueron captados de entre las personas que acudían a realizar gestiones a un centro administrativo; en la puerta de dicho centro se les solicitaba su participación altruista en el estudio y se le pedía cumplimentar un cuestionario en formato electrónico dándoles la información necesaria para que pudieran acceder al formulario a través de redes sociales. Cuando se accedía al cuestionario electrónico, se ofrecía información sobre el tema y los objetivos del estudio y se solicitaba el consentimiento marcando la casilla correspondiente; cuando se marcaba la casilla NO, el formulario ofrecía un mensaje de agradecimiento y se salía del programa.

El resto de los participantes $\left(n_{2}\right)$ fueron algunos residentes de un centro de atención a personas con trastorno mental crónico. A través de los medios habituales en el 
centro (tablón de anuncios) se ofreció a todos los residentes la posibilidad de participar en el Programa de promoción de la salud sexual. Aquellas personas que mostraron su interés fueron convocados a una reunión donde cumplimentaron los cuestionarios en papel. Posteriormente, fueron asignados al azar a dos condiciones: a) asistencia al programa (AP) y b) lista de espera (LE). Los participantes de la condición AP asistieron al programa que se describe más abajo, volviendo a cumplimentar los cuestionarios en la última sesión. A los participantes asignados a la condición LE, se les comunicó que, por razones de espacio, deberían esperar a que finalizara el programa con el grupo AP, y se programaron para ellos actividades culturales y de ocio durante el tiempo que los participantes de la condi- ción AP asistían al programa. Cumplimentaron por segunda vez los cuestionarios 7 semanas después, coincidiendo con el final de programa al que asistieron sus compañeros del grupo AP.

El «Programa piloto de Promoción de la Salud sexual en personas con enfermedad grave» fue diseñado como una actividad participativa y la terapeuta (psicóloga sanitaria) fomentaba en todo momento la comunicación y la implicación activa de los participantes, siempre bajo un prisma de respeto y flexibilidad. En la Tabla 1 se presentan las principales técnicas de educación para la salud sexual que se utilizaron, así como los objetivos que se perseguían con la utilización de cada una de ellas.

Tabla 1. Principales técnicas de educación para la salud sexual utilizadas y objetivos que se pretendían alcanzar

\begin{tabular}{ll}
\hline \multicolumn{1}{c}{ Técnica } & \multicolumn{1}{c}{ Objetivos } \\
\hline Debate & $\begin{array}{l}\text { Mejorar la capacidad de comunicación, escucha, reflexión y argumentación de cada partici- } \\
\text { pante. Además, se pretende fomentar una actitud empática para poder comprender las dife- } \\
\text { rentes posiciones ante una temática determinada. } \\
\text { Role-playing }\end{array}$ \\
Aprendizaje activo, toma de conciencia y comprensión, de todos los participantes del pro- \\
grama, sobre un tema o problema en concreto. \\
Elaboración de materiales \\
Mayéutica
\end{tabular}

El programa se llevó a cabo en 7 sesiones, una sesión semanal de una hora de duración; a estas sesiones asistieron todos los participantes, no produciéndose ninguna baja ni ausencia puntual. La información ofrecida en estas sesiones fue recopilada de López de la Llave y Pérez-Llantada (2011a; 2011b; 2011c), teniendo como propósito el de incrementar el conocimiento de los participantes sobre diferentes aspectos de la salud sexual, la identificación y conocimiento de «los mitos y falacias sobre sexualidad», los aspectos culturales que atribuyen roles estereotipados y fijos a cada género, en relación a su sexo biológico, la respuesta sexual humana, las disfunciones sexuales, los efectos de una sustancia externa (medicamentos u otro tipo de drogas) sobre la sexualidad (respuesta sexual), y los comportamientos de riesgo relacionados con las infecciones de transmisión sexual... Véase Tabla 2 página siguiente.

\section{Análisis de datos}

En primer lugar, se calcularon los valores de algunos estadísticos para describir los resultados (medias y varianzas). Debido a las características de la muestra (ta- maño y procedimiento de extracción) se utilizó la prueba no paramétrica U de Mann-Whitney (muestras independientes) para determinar si había diferencias estadísticamente significativas entre las dos submuestras $\left(n_{1}\right.$ y $\left.n_{2}\right)$. El tamaño del efecto se calculó mediante el estadístico $r$ de Rosenthal (Rosenthal, 2000; Rosnow y Rosenthal, 2003). Se utilizó el mismo procedimiento para las comparaciones entre las condiciones AP y LE. Las comparaciones pre/post en la condición AP se llevaron a cabo mediante el estadístico W de Wilcoxon. Se estableció el 0,05 como nivel de significación.

\section{Resultados}

A continuación, se presentan los resultados obtenidos en relación con los dos objetivos planteados: a) Estudio de las diferencias en conocimientos y actitudes sexuales entre personas con y sin diagnóstico de enfermedad mental y b) Comparaciones entre las condiciones AP y LE, y comparaciones pre/post (condición AP) en conocimientos sobre sexualidad y actitudes sexuales en personas con un diagnóstico de enfermedad mental. 
Tabla 2. Distribución de frecuencias de diagnósticos clínicos de los participantes de la muestra clínica $(n=50)$

\begin{tabular}{|c|c|c|c|}
\hline Sesión & Objetivo & Actividades & Materiales \\
\hline 1 & Toma de contacto con el grupo & Evaluación inicial & $\begin{array}{l}\mathrm{CCS} \\
\mathrm{COS}\end{array}$ \\
\hline 2 & $\begin{array}{l}\text { Mejora de la conciencia corporal e infor- } \\
\text { mación sobre el proceso de sexuación }\end{array}$ & $\begin{array}{l}\text { Exposición de las expectativas de los parti- } \\
\text { cipantes } \\
\text { Explicación de la importancia de nuestro } \\
\text { cuerpo en relación a la sexualidad } \\
\text { Debate sobre cómo los hombres y mujeres } \\
\text { son educados } \\
\text { Explicación sobre la relación de la vivencia } \\
\text { de nuestro cuerpo e imagen corporal con el } \\
\text { desarrollo de la autoestima }\end{array}$ & Presentaciones en Powert Point \\
\hline 3 & $\begin{array}{l}\text { Ofrecer conocimientos dobre la respuesta } \\
\text { sexual humana y disfunciones sexuales }\end{array}$ & $\begin{array}{l}\text { Explicación de la respuesta sexual y sus res- } \\
\text { pectivas fases } \\
\text { Información sobre las disfunciones sexua- } \\
\text { les recogidas en el DSM } 5 \text {. }\end{array}$ & Láminas ilustrativas \\
\hline 4 & Desmitificar algunas falacias sexuales & $\begin{array}{l}\text { Método socrático para favorecer el debate y } \\
\text { desmitificar las falsas creencias en relación } \\
\text { a la Sexualidad. }\end{array}$ & Presentaciones en Powert Point \\
\hline 5 & $\begin{array}{l}\text { Ofrecer información sobre medicamentos } \\
\text { y/o sustancias que afectan a la respuesta se- } \\
\text { xual }\end{array}$ & $\begin{array}{l}\text { Información relativa a los efectos de sus- } \\
\text { tancias externas sobre la respuesta sexual. }\end{array}$ & Presentaciones en Powert Point \\
\hline 6 & $\begin{array}{l}\text { Ofrecer información métodos anticoncepti- } \\
\text { vos }\end{array}$ & $\begin{array}{l}\text { Explicación del término «anticoncepción» } \\
\text { Ventajas e inconvenientes de los diferentes } \\
\text { métodos anticonceptivos. }\end{array}$ & Presentaciones Powert Point \\
\hline 7 & $\begin{array}{l}\text { Ofrecer información infecciones de trans- } \\
\text { misión sexual y evaluacion final }\end{array}$ & $\begin{array}{l}\text { Información sobre las Infecciones de Trans- } \\
\text { misión Sexual } \\
\text { Evaluación final }\end{array}$ & $\begin{array}{l}\mathrm{CCS} \\
\mathrm{COS}\end{array}$ \\
\hline
\end{tabular}

Nota. COS = Cuestionario de opiniones sobre la sexualidad; $\mathrm{CCS}=$ Cuestionario sobre conocimientos de Sexualidad.

a) Comparaciones entre personas sin diagnóstico de enfermedad mental (PSDEM, $n_{1}=30$ ) y personas con diagnóstico de enfermedad (PCDEM, $n_{2}=23$ ).

La Tabla 3 muestra las medias y desviaciones típicas de las dos variables consideradas (conocimientos y acti- tudes sexuales) de la muestra total $(N=53)$ y para cada una de las variables de las dos submuestras (PSDEM = 30 y PCDEM = 23).

Mediante los contrastes realizados utilizando el estadístico U de Mann-Whitney se encontraron diferencias

Tabla 3. Medias, desviaciones típicas y puntuaciones máxima y mínima, de las variables consideradas, en las dos muestras estudiadas

\begin{tabular}{|c|c|c|c|c|c|}
\hline & Grupo & Media & Desviación típica & Mínimo & Máximo \\
\hline \multirow{3}{*}{$\begin{array}{l}\text { Conocimientos sobre sexua- } \\
\text { lidad (CCS) }\end{array}$} & Total $(N=53)$ & 8.27 & 1.67 & 1.33 & 10.00 \\
\hline & $\operatorname{PCDEM}\left(n_{2}=23\right)$ & 7.11 & 1.88 & 1.33 & 9.65 \\
\hline & $\operatorname{PSDEM}\left(n_{1}=30\right)$ & 9.15 & 0.65 & 7.67 & 10.00 \\
\hline \multirow[t]{3}{*}{ Actitudes sexuales (COS) } & Total $(N=53)$ & 2.10 & 0.66 & 1.08 & 3.18 \\
\hline & $\operatorname{PCDEM}\left(n_{2}=23\right)$ & 1.89 & 0.73 & 1.08 & 3.18 \\
\hline & $\operatorname{PSDEM}\left(n_{1}=30\right)$ & 2.26 & 0.55 & 1.15 & 3.15 \\
\hline
\end{tabular}

Nota. PCDEM = Personas con diagnóstico de enfermedad mental; PSDEM = Personas sin diagnóstico de enfermedad mental; $\mathrm{COS}=$ Cuestionario de opiniones sobre la sexualidad; CCS = Cuestionario sobre conocimientos de Sexualidad. 
estadísticamente significativas entre ambos grupos en cuanto a conocimientos sobre sexualidad (la media de rangos del grupo de PCDEM $(n=23)$ y del grupo de PSDEM $(n=30)$ fueron 15.61 y 35.73 respectivamente; $U=83.22$ $(Z=-4.742), p=.000)$ con un tamaño del efecto, en términos de Cohen (1968), grande $r=.651$ ). También fueron estadísticamente significativas las diferencias en cuanto a las actitudes sexuales sobre sexualidad (la media de rangos del grupo de PCDEM $(n=23)$ y del grupo de PS-
DEM $(n=30)$ fueron 21.78 y 31.00 respectivamente; $U=225(Z=-2.158), p=.031)$; en este caso el tamaño del efecto, en términos de Cohen (1968), se considera pequeño $(r=.0, .296)$. En la Figura 1 se muestra una representación de estos resultados.

b) Comparaciones entre las condiciones del grupo $n_{2}$ : AP-LE (Tabla 4 y Figura 1 ) y comparaciones pre/post (condición AP) en conocimientos sobre sexualidad y actitudes sexuales (Tabla 5 y Figura 2).

Tabla 4. Resultados antes y después de la aplicación del programa. Medias, desviaciones típicas de las variables consideradas, en las dos condiciones del grupo de PCDEM (AP y LE)

\begin{tabular}{lccc}
\hline & & Pretratamiento & Postratamiento \\
\cline { 2 - 4 } & Condición & Media $(D T)$ & Media $(D T)$ \\
\hline Conocimientos sobre sexualidad (CCS) & AP $(n=13)$ & $7.58(1.56)$ & $9.22(0.95)$ \\
Actitudes sexuales $(\mathrm{COS})$ & & $6.16(2.17)$ & $6.48(2.02)$ \\
& LE $(n=10)$ & $1.98(0.78)$ & $1.29(0.37)$ \\
& AP $(n=13)$ & $1.79(0.69)$ & $1.75(0.60)$ \\
\hline
\end{tabular}

Nota $. \mathrm{AP}=$ Asistencia al programa; $\mathrm{LE}=$ Lista de espera; $\mathrm{COS}=$ Cuestionario de opiniones sobre la sexualidad; $\mathrm{CCS}=\mathrm{Cuestionario}$ sobre conocimientos de Sexualidad.

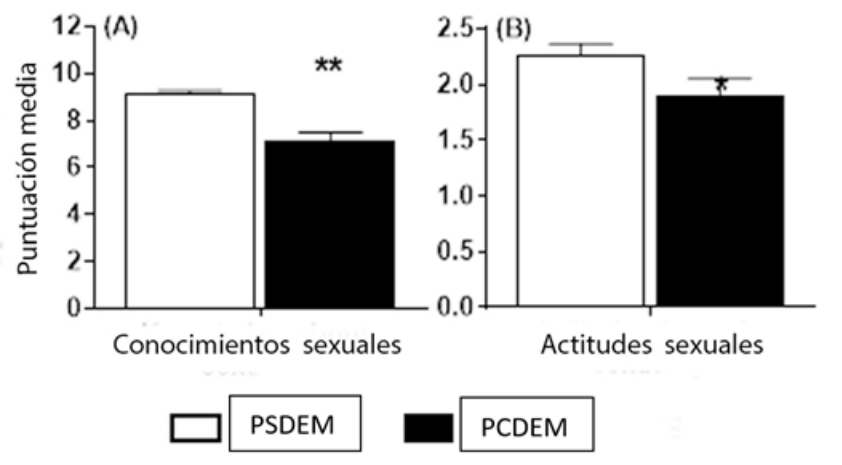

Figura 1. Representación de los estadísticos de tendencia central y variabilidad de las puntuaciones sobre (A) conocimientos sobre sexualidad y (B) actitudes hacia el sexo en personas sin diagnóstico de enfermedad mental (PSDEM, $n_{1}=30$ ) y personas con diagnóstico de enfermedad (PCDEM, $n_{2}=23$ ).

En las medidas tomadas antes de la aplicación del programa, los contrastes realizados con el estadístico U Mann-Whitney mostraron ausencia de diferencias estadísticamente significativas entre ambas condiciones de este grupo para las dos variables consideradas: conocimientos sobre sexualidad y actitudes sexuales. En cuanto a conocimientos sobre sexualidad (la media de rangos del grupo de AP $(n=13)$ y del grupo LE $(n=10)$ fueron 13.31 y 10.30 respectivamente; $U=55.00(Z=-0.622)$, ns) con un tamaño del efecto pequeño $(r=.129)$. Tampoco fueron estadís- ticamente significativas las diferencias en actitudes sexuales sobre sexualidad (la media de rangos del grupo de AP $(n=13)$ y del grupo LE $(n=10)$ fueron 12.77 y 11.00 respectivamente; $U=48.00(Z=-1.058)$, ns $)$ con un tamaño del efecto pequeño $(r=.220)$

Tras la aplicación del programa, los contrastes realizados con el estadístico U de Mann-Whitney mostraron diferencias estadísticamente significativas entre ambos grupos en cuanto a conocimientos sobre sexualidad (la media de rangos del grupo de AP $(n=13)$ y del grupo LE $(n=10)$ fueron 15.85 y 7.00 respectivamente; $U=15.00$ $(Z=-3.106), p=.002)$ con un tamaño del efecto grande $(r=.648)$. Las diferencias en actitudes sexuales también fueron estadísticamente significativas (la media de rangos del grupo de AP $(n=13)$ y del grupo LE $(n=10)$ fueron 9.31 y 15.50 respectivamente; $U=30.00(Z=-2.173)$, $p=.03)$ con un tamaño del efecto mediano $(r=.453)$.

También se encontraron diferencias estadísticamente significativas en los resultados entre las condiciones del grupo AP (antes y después del programa de intervención). Los conocimientos sobre sexualidad se incrementaron entre las mediciones efectuadas antes y después de implementar el Programa piloto de Promoción de la salud sexual $(Z=-3.184 ; p=.001)$. Los resultados en actitudes sexuales señalan una tendencia más liberal $(Z=-3.180 ; p=.001)$. Véase Tabla 5 y la Figura 2. 
Tabla 5. Comparación PRE/POST mediante prueba de rangos con signos de Wilcoxon

\begin{tabular}{lcccc}
\hline & Rangos & Valor rango & Rango promedio & Suma de rangos \\
\hline & negativos & 0 & 0.00 & 0.00 \\
PRE/POST & positivos & 13 & 7.00 & 91.00 \\
$\begin{array}{l}\text { Conocimientos sobre se- } \\
\text { xualidad (CCS) }\end{array}$ & Empates & 0 & & $7.18^{*}$ \\
& negativos & 12 & 6.50 & 0.00 \\
PRE/POST & positivos & 0 & 0.00 & $-3.18^{*}$ \\
Actitudes sexuales (COS) & Empates & 1 & & 0.00 \\
\hline
\end{tabular}

Nota. COS $=$ Cuestionario de opiniones sobre la sexualidad; $\mathrm{CCS}=$ Cuestionario sobre conocimientos de Sexualidad. $*=p<.01$.

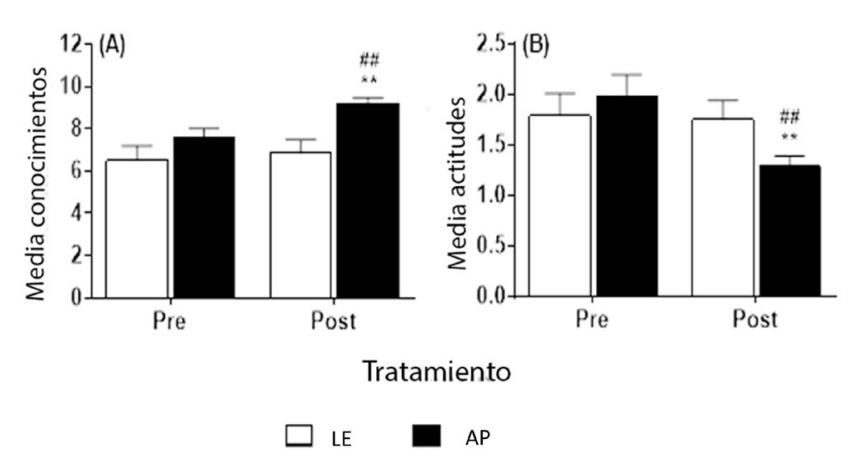

Figura 2. Representación de los estadísticos de tendencia central y variabilidad de las puntuaciones sobre (A) conocimientos sobre sexualidad y (B) actitudes hacia el sexo en pacientes con diagnóstico de enfermedad mental a los que se aplicó el tratamiento mediante el programa de intervención (Asistencia al programa, AP, $n=13$ ) y aquellos que no recibieron la intervención (Lista de espera, LE, $n=10$ ). \#\# $=p<.05$ al comparar los grupos experimental y control. $* *=p<.01$ al comparar al grupo experimental antes y después del programa de intervención.

\section{Discusión}

El primer objetivo de este estudio era conocer si existían diferencias, en conocimientos sobre sexualidad y en actitudes sexuales, en personas provenientes de poblaciones diferentes (unas con diagnóstico en enfermedad mental y otras sin esta condición). Los resultados mostraron que las personas que no tienen una enfermedad mental asociada obtuvieron puntuaciones más altas en conocimientos sobre sexualidad. Estos datos podrían sustentarse en diversas explicaciones. Por un lado, es importante señalar la afectación del estigma en las personas con malestar psíquico, confirmando que tal estigma afecta a los conocimientos y comportamientos sexuales (Bonfils et al., 2015). Por otro lado, la escasez de programas promoción de la salud sexual que se implementan en esta población puede contribuir a que estas personas sean privadas de recibir información rigurosa y actualizada sobre estos asuntos, y con ello mantener una información inadecuada o desactualizada sobre sexualidad.

En cuanto a las actitudes sexuales, las personas con enfermedad mental mostraron menos perjuicios en relación con la sexualidad, pudiéndose describir sus actitudes sexuales como más liberales o erotofílicas. Este resultado parece coherente con la trayectoria de estas personas por los diversos recursos de salud mental. En estos servicios de salud mental, visitan de manera reiterada a profesionales que, muchas veces, prestan atención a la sexualidad, lo que puede fomentar actitudes sexuales más saludables y positivas. No obstante, esta consideración, debería ser corroborada en investigaciones futuras.

Los resultados respecto a estas diferencias en actitudes sexuales entre población general y personas con enfermedad mental no parecen de tal magnitud (tamaño del efecto pequeño, en términos de Cohen, 1968), que justifiquen la necesidad de diseñar e implantar programas de promoción de la salud sexual netamente diferentes para ambos tipos de poblaciones. Las diferencias en conocimientos muestran diferencias más elevadas. En este sentido, probablemente las personas con enfermedad mental se podrían beneficiar de las mismas intervenciones de promoción de la salud sexual que se diseñan para ser implantadas en adultos pertenecientes a la población general, aunque convendría en su caso reforzar lo relativo a la mejora de sus conocimientos sobre sexualidad.

En líneas generales, en relación con el segundo objetivo de nuestro estudio, parece que la intervención mediante un Programa Piloto de Promoción de Salud sexual contribuyó a mejorar los conocimientos sobre sexualidad de los participantes en el programa. Además, propició el desarrollo de actitudes sexuales liberales o positivas. Estos datos apoyan la idea de partida sobre la utilidad de estas acciones de promoción de la salud sexual en estos colectivos. 
Los 13 participantes del grupo que asistieron al programa mostraron incrementos en su nivel de conocimientos, así como un desplazamiento de sus actitudes sexuales hacia el polo más positivo o saludable. Este hecho, están en línea con la idea de que los programas diseñados para tal fin son una buena estrategia para promover la salud sexual en este colectivo (Higgins et al., 2006). Asimismo, un resultado destacado fue que, al incluir unos contenidos más extensos, que los que vienen siendo habituales en los programas de promoción de la salud sexual que se aplican en este contexto, nuestro programa obtuvo unos resultados favorables. Este dato, confirma la idea de que resulta provechoso ampliar el contenido y los objetivos de los programas de salud sexual dirigidos a esta población, que tradicionalmente se centraban solamente en dos áreas: las disfunciones sexuales causadas por los psicofármacos y las infecciones de transmisión sexual (Higgins et al., 2006).

Los resultados obtenidos pueden contemplarse como un punto de partida que permita impulsar la implantación de este tipo de programas de promoción de la salud sexual en las personas con enfermedad mental. En este sentido esta reflexión concuerda con lo expuesto por Navarro et al. (2003), que señalan la importancia de la vida sexual para estas personas.

De acuerdo con Pandor et al. (2015), se puede considerar como una necesidad urgente el continuar implantando y evaluando, de forma rigurosa, programas de promoción de la salud sexual en personas con enfermedad mental grave. Para ello nos parece importante que los profesionales de la salud mental superen las barreras de la sintomatología inherente a esta enfermedad y amplíen el campo de actuación a otras dimensiones fundamentales en la vida de estas personas.

Es necesario destacar que este estudio ofrece los resultados de un programa piloto que se aplicó para evaluar la conveniencia, posible utilidad y eficacia de este tipo de intervenciones sobre la salud sexual de las personas con enfermedad mental grave y prolongada. Esto podría considerarse como una limitación metodológica en cuanto a la posible generalización de nuestros resultados, debido principalmente al procedimiento para la selección de la muestra de participantes, lo que resulta determinante en el control de variables extrañas.

Por otro lado, queremos señalar la escasa atención que se ha prestado a esta cuestión: la sexualidad, en el ámbito de la trastorno mental crónico. Esto se manifiesta en los pocos informes sobre este tipo de actuaciones de los profesionales de la salud. Hoy resulta obvio que las actuaciones de este tipo, en este medio, son determinantes para conseguir mejores niveles de salud y bienestar entre los ciudadanos que padecen estas enfermedades (véase por ejemplo Pandor et al., 2015). Es por eso por lo que se hace necesario, desde nuestro punto de vista, impulsar, no solo las actuaciones de promoción de la salud sexual en esta población, sino que, además, estas actuaciones sean evaluadas en cuanto a su eficacia. En este sentido, este trabajo exploratorio, pretende contribuir a abrir las puertas a este tipo de actuaciones sanitarias y promover la conciencia de la necesidad de evaluar estos programas.

Como señalábamos al comienzo de este apartado, dados los objetivos netamente exploratorios de este estudio, no resulta conveniente la generalización de estos resultados más allá del ámbito en que se aplicó este programa piloto de promoción de la salud sexual.

\section{Conflictos de intereses}

Los autores declaran no tener conflictos de intereses.

\section{Referencias}

American Psychiatric Association (2013). Diagnostic and statistical manual of mental disorders (5th ed.). https://doi.org/10.1176/ appi.books.9780890425596

Baldwin, D. S. \& Foong, T. (2013). Antidepressant drugs and sexual dysfunction. British Journal of Psychiatry, 202(6), 396-397. https://doi.org/10.1192/bjp.bp.112.110650

Blanc, A. (2020). El papel mediador de las actitudes entre los comportamientos sexuales de hombres y mujeres. Revista Internacional de Andrología, 19(2), 107-111. https://doi.org/ 10.1016/j.androl.2019.11.002

Bonfils, K. A., Firmin, R. L., Salyers, M. P. \& Wright, E. R. (2015). Sexuality and intimacy among people living with serious mental illnesses: Factors contributing to sexual activity. Psychiatric Rehabilitation Journal, 38(3), 249-255. https://doi.org/10.1037/ prj0000117

Brown, L. K., Hadley, W., Stewart, A., Lescano, C. L., Whiteley, L., Donenberg, G., \& DiClemente, R. (2010). Psychiatric disorders and sexual risk among adolescents in mental health treatment. Journal of Consulting and Clinical Psychology, 78(4), 590-597. https://doi.org/10.1037/a0019632

Carrera, M. V., Lameiras, M., Foltz, M., Mangana, A. M. y Rodríguez, Y. (2007). Evaluación de un programa de educación sexual con estudiantes de Educación Secundaria Obligatoria. International Journal of Clinical and Health Psychology, 7(3), 739-751. Disponible en: https://www.redalyc.org/articulo.oa?id=33770311

Corbett, R., Elsom, S., Sands, N. \& Prematunga, R. (2017). An exploratory investigation of sexual health screening in the first 12 weeks of case management in populations with severe mental illness. International Journal of Mental Health Nursing, 26(2), 160-169. https://doi.org/10.1111/inm.12257

Costa, M., y López, E. (1996). Educación para la salud. Una estrategia para cambiar los estilos de vida. Ediciones Pirámide.

Diéguez, J. L., López Castedo, A., Sueiro, E. y López, F. (2005). Propiedades psicométricas de la escala de actitudes hacia la sexualidad (ATSS) ampliada. Cuadernos de Medicina 
Psicosomática y Psiquiatría de Enlace, 74, 46-56. Disponible en: https://dialnet.unirioja.es/servlet/articulo?codigo=1368867

Fishbein, M. \& Ajzen, I. (2011). Predicting and changing behavior: The reasoned action approach. 1st Ed. Taylor \& Francis.

Fisher, T. D. \& Hall, R. G. (1988). A Scale for the Comparison of the Sexual Attitudes of Adolescents and their Parents. Journal of Sex Research, 24, 90-100. https://doi.org/10.1080/ 00224498809551400

Fisher, W. A., Byrne, D., White, L. A. \& Kelley, K. (1988). Erotophobia-erotophilia as a dimension of personality. Journal of Sex Research, 25, 123-151. https://doi.org/10.1080/ 00224498809551448

Ford, E., Rosenberg, M., Holsten, M. \& Boudreaux, T. (2003). Managing sexual behavior on adult acute care inpatient psychiatric units. Psychiatric Services, 54, 346-350. https:// doi.org/10.1176/appi.ps.54.3.346

García, M. y Suárez, O. (2007). Programa de Educación Sexual para los Centros de Menores de Asturias. Consejería de Vivienda y Bienestar Social. Gobierno del Principado de Asturias. https:// observatoriodelainfanciadeasturias.es/documentos/ programasexualmenores.pdf

Goldman, H., Gattozzi, A. \& Taube, C. A. (1981). Defining and counting the chronically mentally ill. Hosp Community Psychiatry, 32(1), 22-22. https://doi.org/10.1176/ps.32.1.21

Higgins, A., Barker, P. \& Begley, C. M. (2006). Sexual health education for people with mental health problems: what can we learn from the literature? Journal of Psychiatric and Mental Health Nurses, 13 (6), 687-697. https://doi.org/10.1111/ j.1365-2850.2006.01016.x

Huguelet, P., Mohr, S., Miserez, C., Castellano, P., Lutz, C., Boucherie, M., Yaron, M., Perroud, N. \& Bianchi F. (2015). An Exploration of Sexual Desire and Sexual Activities of Women with Psychosis. Community Mental Health Journal 51, 229-238. https://doi.org/10.1007/s10597-014-9768-x

Kaltenthaler, E., Pandor, A. \& Wong, R. (2014). The effectiveness of sexual health interventions for people with severe mental illness: a systematic review. Health technology assessment, 18(1), 1-74. https://doi.org/10.3310/hta18010

Khan, M. R., Kaufman, J. S., Pence, B. W., Gaynes, B. N., Adimora, A. A., Weir, S. S. \& Miller, W. C. (2009). Depression, sexually transmitted infection, and sexual risk behavior among young adults in the United States. Archives of Pediatric and Adolescent Medicine, 163 (7), 644-652. https:// doi.org/10.1001/archpediatrics.2009.95

Kirby, D. (2011) The impact of sex education on the sexual behaviour of young people. United Nations. Department of Economic and Social Affairs. Population Division. Expert Paper No. 2011/12 https://www.un.org/en/development/desa/population/ publications/pdf/expert/2011-12_Kirby_Expert-Paper.pdf

Landry, D. J., Darroch, J. E., Singh, S. \& Higgins, J. (2003) Factors associated with the content of sex education in U.S. public secondary schools. Perspect Sex Reprod Health, 35(6), 261-9. https://doi.org/10.1363/psrh.35.261.03.

López de la Llave, A. (2016). Sexualidad positiva. En A. López de la Llave (Ed.): Sexología positiva: Placer, salud y bienestar. Universidad Nacional de Educación a Distancia.

López de la Llave, A. (2019). Psicología y Salud sexual. En J. M. Buceta y B. Más (Ed.): Psicología y Salud: Evaluación y tratamiento. Dykinson.
López de la Llave, A., y Pérez-Llantada, M.C. (2011a). Respuesta sexual humana. Anatomía y fisiología. [Archivo pdf]. https:// www2.uned.es/saludsexual/apuntes/respuestasexual.pdf

López de la Llave, A., y Pérez-Llantada, M.C. (2011b). Actitudes sexuales saludables. Falacias Sexuales. [Archivo pdf]. https:// www2.uned.es/saludsexual/apuntes/actitudessexuales.pdf

López de la Llave, A., y Pérez-Llantada, M.C. (2011c). Educación para la Salud sexual en la infancia y en la adolescencia. [Archivo pdf]. https://www2.uned.es/saludsexual/apuntes/ educacionsexual.pdf.

López de la Llave, A., Pérez-Llantada, M. C., Placer P. y Flores, Y. (2015). La promoción de la salud sexual en contextos informales y su relación con los conocimientos y las actitudes sexuales de las mujeres. Revista de Psicopatología y Psicología Clínica, 20, 209-218. https://doi.org/10.5944/rppc. vol.20.num.3.2015.15896

McCann, E. (2010). The sexual and relationship needs of people who experience psychosis: Quantitative findings of a UK study. Journal of Psychiatric and Mental Health Nurses, 17, 295-303. https://doi.org/10.1111/j.1365-2850.2009.01522.x

Meade, C. S. (2006). Sexual risk behavior among persons dually diagnosed with severe mental illness and substance use disorder. Journal of Substance Abuse Treatment, 30(2), 147157. https://doi.org/10.1016/j.jsat.2005.11.005

Morales, A., Espada, J. P., Orgilés, M., Escribano, S., Johnson, B. T. \& Lightfoot M. (2018) Interventions to reduce risk for sexually transmitted infections in adolescents: A metaanalysis of trials, 2008-2016. PLOS ONE, 13(6), e0199421. https://doi.org/10.1371/journal.pone.0199421

Navarro, F.M., Vega, F.M., y Montejo, A.L. (2003). La sexualidad en la esquizofrenia. En A.L. Montejo (Ed.), Sexualidad y salud mental (pp. 117-128). Glosa.

Organización Mundial de la Salud (septiembre 2020). Temas de salud: Salud sexual. https://www.who.int/topics/sexual_health/es/

Organización Panamericana de la Salud/Organización Mundial de la Salud. (2000). Promoción de la Salud Sexual. Recomendaciones para la acción. Guatemala. http://www.paho.org/spanish/hcp/ hca/salud_sexual.pdf.

Pandor, A., Kaltenthaler, E., Higgins, A., Lorimer, K., Smith, S., Wylie, K. \& Wong, R. (2015). Sexual health risk reduction interventions for people with severe mental illness: a systematic review. BMC Public Health, 15(1),138. https://doi. org/10.1186/s12889-015-1448-4

Pérez, M. J., Echauri, M., Ancizu, E. y Chocarro, J. (2006). Manual de Educación para la Salud. Instituto de Salud Pública. Sección de Promoción de Salud. Gobierno de Navarra. https://www. navarra.es/nr/rdonlyres/049b3858-f993-4b2f-9e332002e652eba2/194026/manualdeeducacionparalasalud.pdf

Pérez-Conchillo, M. (2019). Oportunidades y retos para los Derechos sexuales en la era digital. En A. Giami \& E. Janssen: Abstracts for the 24th Congress of the World Association for Sexual Health (WAS). International Journal of Sexual Health, 31, 1-627. https://doi.org/10.1080/19317611.2019.1661941

Raisi, F., Yahyavi, S., Mirsepassi, Z., Firoozikhojastefar, R. \& Shahvari, Z. (2018). Neglected sexual needs: A qualitative study in Iranian patients with severe mental illness. Perspectives in Psychiatric Care, 54(4), 488-494. https://doi.org/10.1111/ppc.12253

Rosenthal, R. (2000). Effect sizes in behavioral and biomedical research: Estimation and interpretation. En L. Bickman (Ed.), 
Validity and social experimentation (Vol. 1, pp. 121-139). Donald Campbell's legacy.

Rosnow, R. L. \& Rosenthal, R. (2003). Effect sizes for experimenting psychologist. Canadian Journal of Experimental Psychology, 57(3), 221-237. https://doi.org/10.1037/h0087427

Ruiz Sternberg, A. M., Latorre Santos, C., Beltran Rodriguez, J., Ruiz Sternberg, J. y Velez Van Meerbeke, A. (2005). Conocimientos, actitudes y prácticas en salud sexual y reproductiva en una población universitaria. [Archivo pdf]. http://www.cemera.cl/ sogia/pdf/2005/XII3conocimientos.pdf

Serretti, A. \& Chiesa, A. (2011). A meta-analysis of sexual dysfunction in psychiatric patients taking antipsychotics. International Clinical Psychopharmacology, 26(6), 130-140. https://doi.org/10.1097/YIC.0b013e328341e434

Susser, E., Valencia, E. \& Conover, S. (1993). Prevalence of HIV infection among psychiatric patients in a New York City men's shelter. American Journal of Public Health, 83, 568-570. https://doi.org/10.2105/AJPH.83.4.568

Susser, E., Valencia, E., Miller, M., Tsai, W. Y., Meyer-Bahlburg, H. \& Conover, S. (1995). Sexual behavior of homeless mentally ill men at risk for HIV. American Journal of Psychiatry, 152(4), 583-587. https://doi.org/10.1176/ajp.152.4.583

Test, M. A. \& Berlin. S. B. (1981). Issues of special concern to chronically mentally ill women. Professional Psycholgy: Research and Practice, 12(1), 136-145. https:// doi.org/10.1037/07357028.12.1.136
Wainberg, M. L., González, M. A., McKinnon, K., Elkington, K. S., Pinto, D., Mann, C. G. \& Mattos, P. E. (2007). Targeted ethnography as a critical step to inform cultural adaptations of HIV prevention interventions for adults with severe mental illness. Social Science Medicine, 65(2), 296-308. 2007. http:// doi.org/10.1016/j.socscimed.2007.03.020

Walsh, C., McCann, E., Gilbody, S. \& Hughes, E. (2014). Promoting HIV and sexual safety behaviour in people with severe mental illness: A systematic review of behavioural interventions. International Journal of Mental Health Nursing, 23(4), 344-354. 2014. http://doi.org/10.1111/inm.12065

Werner, S. (2012). Needs assessment of individuals with serious mental illness: Can it help in promoting recovery? Community Mental Health Journal, 48, 568-573. http://doi.org/10.1007/ s10597-011-9478-6

Williams, V. S. L., Edin, H. M., Hogue, S. L., Fehnel, S. E. \& Baldwin, D. S. (2010). Prevalence and impact of antidepressant-associated sexual dysfunction in three European countries: replication in a cross-sectional patient survey. Journal of Psychopharmacology, 24(4), 489-496. https://doi.org/10.1177/0269881109102779

Wright, E. R., Wright, D. E., Perry, B. L. \& Foote-Ardah, C. E. (2007). Stigma and the sexual isolation of people with serious mental illness. Social Problem, 54(1), 78-98. https://doi. org/10.1525/sp.2007.54.1.78 


\section{Apéndice A}

Ítems del Cuestionario sobre Conocimientos de Sexualidad (CCS).

- La vida sexual de una mujer continua con la menopausia.

- La masturbación, tanto femenina como masculina, causa daños a las personas que lo practican.

- Las personas con enfermedad mental no deben tener actividad sexual.

- Las relaciones sexuales son sinónimo de coito.

- El tamaño del pene no interfiere con el placer que proporciona.

- Un verdadero hombre debe estar siempre listo para tener sexo.

- La próstata y el útero son órganos sexuales internos, el primero masculino el segundo femenino.

- El orgasmo es la fase más intensa, pero de menos duración.

- El orgasmo únicamente se puede alcanzar a través de la penetración.

- La ansiedad o el estrés NO afectan a la respuesta sexual.

- La existencia de sentimientos negativos hacia uno mismo, los deterioros en la autoimagen o que la persona no se encuentre a si misma suficientemente atractiva, suelen afectar al deseo sexual.

- Las personas que sufren problemas sexuales, no es bueno que acudan a un especialista, el problema se resolverá solo.

- El alcohol y la marihuana aumentan la potencia sexual.

- Las drogas son enemigos para la respuesta sexual, el crecimiento erótico y el intercambio amoroso.

- La cocaína nunca provoca trastornos sexuales, como la disfunción eréctil o priapismo.

- Los fármacos psiquiátricos no afectan nunca a la respuesta sexual.

- Una persona que su medicación le afecta a su respuesta sexual, deberá comunicarlo a un especialista que le ayude en su problemática.

- La única solución ante la problemática sexual producida por la medicación, es dejar de tomar esa misma medicación.

- El preservativo, tanto masculino como femenino, NO evita ninguna infección de transmisión sexual.

- Las Infecciones de Transmisión Sexual, nunca se transmiten si la mujer tiene la menstruación.

- Las personas seropositivas pueden transmitir el virus del sida, aunque no estén enfermas.

- Ninguna infección de transmisión sexual tiene tratamiento.

- Es posible saber si hay infección por el VIH al día siguiente de una práctica de riesgo.

- Las píldoras anticonceptivas son un método eficaz para prevenir la transmisión del VIH en relaciones sexuales con penetración.

- Los anticonceptivos de emergencia se pueden usar de manera habitual.

- La píldora anticonceptiva es un método hormonal.

- Cualquier método anticonceptivo que es eficaz para prevenir el embarazo, lo será también para evitar las Infecciones de Transmisión Sexual.

— La «marcha atrás» tiene un alto porcentaje de éxito tanto para evitar embarazos como para evitar Infecciones de transmisión sexual.

- La comunicación entre personas que van a mantener relaciones sexuales es importante para llevar conductas de sexo seguro.

- Tomarse la temperatura basal es un método eficaz para la evitación de embarazos e Infecciones de Transmisión Sexual.

\section{Apéndice B}

Enunciados del Cuestionario de Opiniones sobre la Sexualidad (COS)

- Se debería dar información sobre anticoncepción a todas aquellas personas que vayan a tener una conducta coital.

- Los tocamientos o caricias que estimulan alguna parte de nuestro cuerpo, o el cuerpo completamente, constituyen una conducta inmoral, a no ser que se esté casado con esa persona.

- Con la edad (ej.: con la llegada de la menopausia en las mujeres), se deja de tener la capacidad de tener orgasmos y disfrute del sexo.

- Una persona contagiada con una infección de transmisión sexual tiene lo que se merece.

- La educación sexual es necesaria tanto en contextos informales (casa, amigos...), como en contextos formales (la escuela).

- Si una persona se masturba, puede tener graves consecuencias.

- Una mujer o un hombre que no alcancen siempre el orgasmo, tienen problemas sexuales, el hombre no es un verdadero hombre y la mujer es una frígida.

- La conducta homosexual es una forma aceptable de preferencia sexual.

- La educación sexual es muy peligrosa.

- Tener fantasías sexuales constituyen un pensamiento inmoral.

- Usar métodos anticonceptivos, no es adecuado en relaciones de larga duración.

- Las mujeres son las que deben estar más a cargo de los hijos.

- Es más adecuado que sea el hombre el que tome la iniciativa en las relaciones sexuales. 